\title{
A united statement of the global chiropractic research community against the pseudoscientific claim that chiropractic care boosts immunity
}

Pierre Côté ${ }^{1,2,3^{*}}$, André Bussières ${ }^{4,5}$, J. David Cassidy ${ }^{3}$, Jan Hartvigsen ${ }^{6,7}$, Greg N. Kawchuk ${ }^{8}$, Charlotte Leboeuf-Yde ${ }^{9}$, Silvano Mior ${ }^{2,10}$, Michael Schneider ${ }^{11,12}$ and and more than 140 signatories\# call for an end to pseudoscientific claims on the effect of chiropractic care on immune function

\begin{abstract}
Background: In the midst of the coronavirus pandemic, the International Chiropractors Association (ICA) posted reports claiming that chiropractic care can impact the immune system. These claims clash with recommendations from the World Health Organization and World Federation of Chiropractic. We discuss the scientific validity of the claims made in these ICA reports.

Main body: We reviewed the two reports posted by the ICA on their website on March 20 and March 28, 2020. We explored the method used to develop the claim that chiropractic adjustments impact the immune system and discuss the scientific merit of that claim. We provide a response to the ICA reports and explain why this claim lacks scientific credibility and is dangerous to the public. More than 150 researchers from 11 countries reviewed and endorsed our response.
\end{abstract}

Conclusion: In their reports, the ICA provided no valid clinical scientific evidence that chiropractic care can impact the immune system. We call on regulatory authorities and professional leaders to take robust political and regulatory action against those claiming that chiropractic adjustments have a clinical impact on the immune system.

Keywords: Chiropractic, Spinal manipulation, Immunity, Pseudoscience, Coronavirus

\section{Background}

We are currently facing the greatest global public health crisis in a century. Fighting the coronavirus pandemic has required that we change the way we live and observe strict public health guidelines. This is necessary because, at this time, there are no effective vaccines, treatments

\footnotetext{
* Correspondence: pierre.cote@uoit.ca

${ }^{1}$ Faculty of Health Sciences, Ontario Tech University, Oshawa, Canada ${ }^{2}$ Centre for Disability Prevention and Rehabilitation at Ontario Tech University and CMCC, Oshawa, Canada

Full list of author information is available at the end of the article
}

or cures for COVID-19 [1, 2]. Chiropractors, as members of the health care system, should disseminate the best available public health information to the public [3]. Any attempt to behave otherwise can be misleading and potentially dangerous to individual patients and the public at large.

On March 20, 2020, the International Chiropractors Association (ICA), a US based chiropractic organization, posted a report claiming that chiropractic adjustments can boost immune function with the implication that it might be helpful in preventing COVID-19 [4]. In their 
report, the ICA states that: "Although there are no clinical trials to substantiate a direct causal relationship between the chiropractic adjustment and increased protection from the COVID-19 virus, there is a growing body of evidence that there is a relationship between the nervous system and the immune system" and "The observation that those who use chiropractic regularly and do not become ill with cold, flu, or other community shared illnesses is frequent within the profession and should not be ignored" [4]. The ICA position directly contradicts the World Health Organization (WHO) that unequivocally states that "there are no effective health interventions to prevent or treat coronavirus infections" [1, 2], and the World Federation of Chiropractic (WFC) that states that "there is no credible scientific evidence that chiropractic spinal adjustments/manipulations confers or boosts immunity" [3].

On March 28, 2020, the ICA posted a revised report which reiterated the information included in the first report, with the addition of references supporting the link between chiropractic care and immune function [5]. In both reports, the ICA claims that their review of the literature confirms "An association between spinal manipulation and the autonomic nervous system" and that "These studies suggest mechanisms by which spinal influences may mediate a clinically significant impact on immune function." Therefore, the main message of both reports is that chiropractic care can have a clinically meaningful impact on immune system function. We discuss the scientific validity of the claims made by the ICA.

\section{Main body}

We investigated the approach used by the ICA to support their claim that chiropractic adjustments impact the immune system. We compared the ICA claim to the findings and conclusions of one systematic review of the literature on the effect of spinal manual therapies on autonomic nervous system activity [6] and two systematic reviews on the efficacy and effectiveness of chiropractic treatment and manual therapy on the prevention and treatment of non-musculoskeletal disorders [7, 8]. Further, we used a list of warning signs of pseudoscience to assess the scientific merit of the claims [9]. Finally, 153 researchers from 11 countries (8 co-authors and 145 signatories) who are involved in research relevant to chiropractic reviewed and endorsed our response.

While the ICA states "that no claims can be made about COVID-19 and chiropractic", their report implies that chiropractic adjustments can boost the immune system through its effect on the nervous system. The ICA claim rests on two assumptions: i) chiropractic adjustments have a beneficial effect on the nervous system and ii) chiropractic adjustments will improve the immune system through the nervous system. These assumptions are not supported by robust evidence that chiropractic adjustments are efficacious or effective in improving immune function [6-8]. We consider that proclaiming the benefits of chiropractic adjustment/spinal manipulation on immunity during a pandemic is plainly irresponsible and demonstrates a lack of understanding of science, the coronavirus pandemic and public health risks.

Our critical review of the reports suggest that the ICA created a positive narrative for the effect of chiropractic adjustments and immune function report by selectively assembling a series of unconnected basic science studies $[4,5]$. This strategy, called "emphasis on confirmation", is a warning sign of pseudoscience [9]. Moreover, this approach fails to respect the established boundaries that exist between basic and clinical research. For example, two of the basic science studies included in the ICA report were led by one of the signatories of this commentary, Stephen Injeyan DC, PhD [10, 11]. According to Dr. Injeyan: "No published studies have so far demonstrated the clinical significance of spinal manipulation and immune enhancement, our research included. Our studies were conducted in asymptomatic subjects, in vitro cellular models, and the outcomes were measured shortly following SMT. There are no parallels between our experimental research and clinical care." By only citing basic science experiments, the ICA appear to have overlooked the WHO guidance on implementation research, which clearly states that basic science experiments do not provide relevant justification for implementation of a health intervention [12].

Any health care intervention must be evaluated for its clinical efficacy and effectiveness in well-designed randomized controlled trials before it is implemented in clinical practice [12]. This requirement is not new; it was first implemented by the US Food and Drug Administration in 1962 [13]. With this in mind, it is all the more noteworthy that none of the studies cited in the ICA report provide evidence that chiropractic adjustments actually prevent the onset of infectious diseases in healthy individuals, or improve the health of patients suffering from a viral infection. We call on the ICA to explain why it does not adhere to internationally accepted standards of research implementation but instead rely on unconnected basic science studies when linking chiropractic care to immune system function.

The ICA also relied on anecdotal evidence to support their claim; this is another warning sign of pseudoscience [9]. For example, the authors state: "The observation that those who use chiropractic regularly and do not become ill with colds, flu, and other community shared illnesses is frequent within the profession and should not be ignored" [4, 5]. At best, this type of anecdotal 
evidence is useful to generate research hypotheses to be tested in high quality randomized clinical trials. To our knowledge, the hypothesis that chiropractic care reduces the risk of becoming ill with viral colds, flu, and other community shared illnesses has never been properly tested. Any claims suggesting otherwise lack scientific merit and should not be used to justify treating patients with chiropractic adjustments.

Advancing extraordinary claims without providing extraordinary evidence should raise significant concerns about the scientific validity of the ICA's position. In their reports, the ICA claims that individuals who received chiropractic care during the 1918 Spanish flu pandemic were 51 to 91 times less likely to die than those who were treated by medical doctors [4, 5]. These effect sizes are too large to be trustworthy and are a red flag of pseudoscience, because extraordinary claims require extraordinary evidence [9]. Using data from a 100-year-old nonpublished, non-randomized controlled trial to suggest that chiropractic adjustments reduces mortality from the flu is scientifically and socially irresponsible.

Pseudoscience has the potential to mislead and misinform at any time; even more so in the midst of a pandemic when the public is vulnerable. The current coronavirus pandemic demands that we act responsibly by adopting sound public health practices as recommended by the WHO [14]. These include but are not restricted to regular handwashing, respiratory etiquette, physical distancing, staying at home, limiting trips outside the home except to obtain food or medicine and wearing a mask if symptomatic [14]. We have seen widespread adherence to the guidance around COVID-19, but as scientists and clinicians we have a public health duty to sound the alarm and denounce pseudoscientific claims such as the ones made by the ICA in its reports.

\section{Conclusion}

We call on regulatory authorities and professional leaders to take appropriate political and regulatory action against those making direct or indirect unsubstantiated claims that spinal adjustments can boost immunity, or benefit patients with infectious diseases, especially coronavirus infections. Above all, these actions must aim to protect the safety and well-being of patients and the public.

\section{Abbreviations}

FDA: Food and Drug Administration; ICA: International Chiropractors Association; WHO: World Health Organization; WFC: World Federation of Chiropractic

Acknowledgements

Not applicable.
\#SIGNATORIES

The views and opinions expressed herein are those solely of the authors and may not represent the views of their institution.

1. Luc Aillet, Private practice, Ghent, Belgium

2. Carlo Ammendolia, University of Toronto, Toronto, Canada

3. Bodil Arnbak, University of Southern Denmark, Odense, Denmark

4. Iben Axen, Karolinska Institute, Stockholm, Sweden

5. Mirjam Baechler; University of Zurich, Zurich, Switzerland

6. Florian Barbier-Cazorla, Institut Franco-Européen de Chiropraxie, Ivry-surSeine, France

7. Gaëtan Barbier, Institut Franco-Européen de Chiropraxie, Toulouse, France

8. Cecilia Bergstrøm, Umeå University, Umeå, Sweden

9. Amber Beynon, Murdoch University, Perth, Australia

10. Marc-André Blanchette, Université du Québec à Trois-Rivières, Trois-

Rivières, Canada

11. Philip S Bolton, University of Newcastle, Callaghan, Australia

12. Alan Breen, AECC University College, Bournemouth, UK

13. Johanne Brinch, University of Southern Denmark and Spine Center of

Southern Denmark, Odense, Denmark

14. Gert Bronfort, University of Minnesota, Minneapolis, USA

15. Benjamin Brown, Macquarie University, Sydney, Australia

16. Paul Bruno, University of Regina, Regina, Canada

17. Mikkel Brunsgaard Konner, University of Southern Denmark and Spine

Center of Southern Denmark, Odense, Denmark

18. Christopher Burrell, Macquarie University, Sydney, Australia

19. Jason W. Busse, McMaster University, Hamilton, Canada

20. David Byfield, Welsh Institute of Chiropractic University of South Wales

Pontypridd, Wales UK

21. Marco Campello, NYU Robert I Grossman School of Medicine. New York, USA.

22. Carol Cancelliere, Ontario Tech University, Oshawa, Canada

23. Linda Carroll, University of Alberta, Edmonton, Canada

24. Christine Cedraschi, University of Geneva, Geneva, Switzerland

25. Charlène Chéron, French Chiropractic Association, Paris, France

26. Ngai Chow, Canadian Memorial Chiropractic College, Toronto, Canada

27. Henrik Wulff Christensen, Nordic Institute of Chiropractic and Clinical

Biomechanics, Odense, Denmark

28. Stine Claussen, University of Southern Denmark, Odense, Denmark

29. Melissa Corso, Ontario Tech University, Oshawa, Canada

30. Matthew A. Davis, University of Michigan Medical School, Ann Arbour, USA

31. Marine Demortier, Institut Franco-Européen de Chiropraxie, Ivry-sur-Seine, France

32. Martin Descarreaux, Université du Québec à Trois-Rivières, Trois-Rivières,

Canada

33. Diana De Carvalho, Memorial University of Newfoundland, St. John's, Canada

34. Katie de Luca, Macquarie University, Sydney, Australia

35. Annemarie de Zoete, Vrije University, Amsterdam, The Netherlands

36. Klaus Doktor, University of Southern Denmark, Odense, Denmark

37. Aron Downie, Macquarie University, Sydney, Australia

38. Alister du Rose, AECC University College, Bournemouth, UK

39. Andreas Eklund, Karolinska Institute, Stockholm, Sweden

40. Roger Engel, Macquarie University, Sydney, Australia

41. Mark Erwin, University of Toronto, Toronto, Canada

42. James E. Eubanks, University of Pittsburgh, Pittsburgh, USA

43. Roni Evans, University of Minnesota, Minneapolis, USA

44. Will Evans, Mississippi State University, Mississippi State, USA

45. Matthew Fernandez, Macquarie University, Sydney, Australia

46. Jonathan Field, University of Southampton, Southampton, UK 47. Gilles

Fournier, Parker Institute, Copenhagen University Hospital at Bispebjerg and Frederiksberg, Denmark

48. Simon French, Macquarie University, Sydney, Australia

49. Signe Fuglkjaer, Private practice, Odense, Denmark

50. Olivier Gagey, University of Paris-Saclay, Orsay, France

51. Rosemary Giuriato, Macquarie University, Sydney, Australia

52. Jordan A. Gliedt, Medical College of Wisconsin, Milwaukee, USA

53. Christine Goertz, Duke University School of Medicine, Durham, USA

54. Guillaume Goncalves, Institut Franco-Européen de Chiropraxie, Ivry-sur-

Seine, France

55. Diane Grondin, Canadian Memorial Chiropractic College, Toronto, Canada

56. Mark Gurden, Royal College of Chiropractors, Reading, UK

57. Mitchell Haas, University of Minnesota, Minneapolis, USA

58. Scott Haldeman, World Spine Care, Los Angeles, USA 
59. Steen Harsted, University of Southern Denmark, Odense, Denmark 60. Lisbeth Hartvigsen, Private Practice, Odense, Denmark

61. Jill Hayden, Dalhousie University, Halifax, Canada

62. Cesar Hincapié, University of Zurich, Zurich, Switzerland

63. Jeffrey J. Hébert, University of New Brunswick, Fredericton, Canada

64. Bue Hesby, University of Southern Denmark, Odense, Denmark

65. Lise Hestbæk, University of Southern Denmark and Nordic Institute of

Chiropractic and Clinical Biomechanics, Odense, Denmark

66. Sheilah Hogg-Johnson, Canadian Memorial Chiropractic College, Toronto,

Canada

67. Maria A. Hondras, University of Kansas Medical Center, Kansas City, USA

68. Margaux Honoré, Institut Franco-Européen de Chiropraxie, Ivry-sur-Seine, France

69. Samuel Howarth, Canadian Memorial Chiropractic College, Toronto,

Canada

70. H. Stephen Injeyan, Canadian Memorial Chiropractic College, Toronto, Canada

71. Stanley Innes, Murdoch University, Perth, Australia

72. Pernille Marie Irgens, University of Oslo, Oslo, Norway

73. Craig Jacobs, Canadian Memorial Chiropractic College, Toronto, Canada

74. Hazel Jenkins, Macquarie University, Sydney, Australia

75. Alan Jenks, Vrije University, Amsterdam, The Netherlands

76. Tue Secher Jensen, University of Southern Denmark and Silkeborg

Regional Hospital, Odense, Denmark

77. Melker Johhansson, University of Southern Denmark, Odense, Denmark

78. Alice Kongsted, University of Southern Denmark and Nordic Institute of Chiropractic and Clinical Biomechanics, Odense, Denmark

79. Deborah Kopansky-Giles, Canadian Memorial Chiropractic College,

Toronto, Canada

80. Rikke Kryger, University of Southern Denmark and Nordic Institute of

Chiropractic and Clinical Biomechanics, Odense, Denmark

81. Arnaud Lardon, Institut Franco-Européen de Chiropraxie, Ivry-sur-Seine, France

82. Henrik Hein Lauridsen, University of Southern Denmark, Odense, Denmark

83. Brent Leininger, University of Minnesota, Minneapolis, USA

84. Nadège Lemeunier, Institut Franco-Européen de Chiropraxie, Toulouse, France

85. Christine Le Scanff, Université Paris-Saclay, Orsay, France

86. Eugene A. Lewis, Duke University School of Medicine, Durham, USA

87. Kathleen Linaker, Western Technical College, La Crosse, USA

88. Lise Lothe, Private Practice, Grimstad, Norway

89. Andrée-Anne Marchand, Université du Québec à Trois-Rivières, Trois-

Rivières, Canada

90. David McNaughton, Macquarie University, Sydney, Australia

91. Anne-Laure Meyer, Institut Franco-Européen de Chiropraxie, Ivry-surSeine, France

92. Peter Miller, AECC University College, Bournemouth, England

93. Anne Mølgaard, University of Southern Denmark, Odense, Denmark

94. Craig Moore, Macquarie University, Sydney, Australia

95. Donald R. Murphy, Brown University, Providence, USA

96. Corrie Myburgh, University of Southern Denmark, Odense, Denmark

97. Birgitte Myhrvold, University of Oslo, Oslo, Norway

98. Dave Newell, AECC University College, Bournemouth, UK 99. Genevieve

Newton, University of Guelph, Guelph, Canada

100. Casper Nim, University of Southern Denmark and Spine Center of

Southern Denmark, Odense, Denmark

101. Margareta Nordin, New York University, New York, USA

102. Luana Nyiro, University of Zurich, Zurich, Switzerland

103. Søren O'Neill, University of Southern Denmark and Spine Center of Southern Denmark, Odense, Denmark

104. Cecilie Øverås, University of Southern Ddenmark, Odense, Denmark

105. Isabelle Pagé, Université du Québec à Trois-Rivières, Trois-Rivières, Canada

106. Mégane Pasquier, Institut Franco-Européen de Chiropraxie, Toulouse, France

107. Charles W. Penza, Miami Veterans Administration Medical Center, Miami, USA

108. Stephen M. Perle, University of Bridgeport, Bridgeport, USA

109. Mathieu Picchiottino, Institut Franco-Européen de Chiropraxie, Ivry-sur-

Seine, France

110. Mathieu Piché, Université du Québec à Trois-Rivières, Trois-Rivières,

Canada
111. Erik Poulsen, University of Southern Denmark, Odense, Denmark 112. Jeffrey Quon, University of British Columbia, Vancouver, Canada 113. Tim Raven, University of Oslo, Oslo, Norway

114. Mana Rezai, Centre for Disability Prevention and Rehabilitation at Ontario Tech University and CMCC Toronto, Canada

115. Eric J. Roseen, Boston University School of Medicine, Boston, MA, USA

116. Sidney Rubinstein, Vrije University, Amsterdam, The Netherlands

117. Louis-Rachid Salmi, Université de Bordeaux, Inserm and CHU de Bordeaux, France

118. Petra Schweinhardt, University of Zurich, Zurich, Switzerland

119. Heather M. Shearer, University of Toronto, Toronto, Canada

120. Laura Sirucek, University of Zurich, Zurich, Switzerland

121. Delphine Sorondo, Institut Franco-Européen de Chiropraxie, Toulouse, France

122. Paula J. Stern, Canadian Memorial Chiropractic College, Toronto, Canada

123. Joel Stevans, University of Pittsburgh, Pittsburgh, USA

124. Mette Jensen Stochkendahl, University of Southern Denmark and Nordic Institute of Chiropractic and Clinical Biomechanics, Odense, Denmark

125. Kent Stuber, Canadian Memorial Chiropractic College, Toronto, Canada

126. Maja Stupar, Canadian Memorial Chiropractic College, Toronto, Canada

127. John Srbely, University of Guelph, Guelph, Canada

128. Michael Swain, Macquarie University, Sydney, Australia

129. Julita Teodorczyk-Injeyan, Canadian Memorial Chiropractic College,

Toronto, Canada

130. Jean Théroux, Murdoch University, Perth, Australia

131. Haymo Thiel, AECC University College, Bournemouth, UK

132. Lars Uhrenholt, University of Aarhus, Aarhus, Denmark

133. Anneke Verbeek, Private practice, Ghent, Belgium

134. Leslie Verville, Ontario Tech University, Oshawa, Canada

135. Karl Vincent, Institut Franco-Européen de Chiropraxie, Ivry-sur-Seine,

France

136. Andrew L. Vitiello, CQUniversity, Sydney, Australia

137. Dan Wang, Ontario Tech University, Oshawa, Canada

138. Kenneth A. Weber, Stanford University School of Medicine, Palo Alto, USA

139. James M. Whedon, Southern California University of Health Sciences,

Whittier, USA

140. Jessica Wong, University of Toronto, Toronto, Canada

141. Francesca Wuytack, Trinity College, Dublin, Ireland

142. James Young, University of Southern Denmark, Odense, Denmark

143. Hainan Yu, Ontario Tech University, Oshawa, Canada

144. Dorte Ziegler, University of Southern Denmark and Spine Center of

Southern Denmark, Odense, Denmark

145. Kristina Boe Dissing, University of Southern Denmark, Odense, Denmark

\section{Authors' contributions}

All authors (Pierre Côté, André Bussières, J. David Cassidy, Jan Hartvigsen, Greg Kawchuk, Charlotte Leboeuf-Yde, Silvano Mior, Mike Schneider) developed, wrote, edited and proofread the commentary. All signatories reviewed the commentary and endorsed its content. The author(s) read and approved the final manuscript.

\section{Funding}

No funding was obtained for this commentary.

\section{Availability of data and materials}

Not applicable.

\section{Ethics approval and consent to participate}

Not applicable.

\section{Consent for publication}

Not applicable.

\section{Competing interests}

PC reports research grants unrelated to this work from Aviva Canada, Canadian Institutes of Health Research-Canada Research Chair Program, Canadian chiropractic Association, Canadian Chiropractic Research Foundation, College of Chiropractors of British Columbia, Et liv i bevegelse" (ELIB), French Chiropractic Association, Financial Services Commission of Ontario, Ontario Ministry of Finance, Ontario Trillium Foundation; travel expenditures unrelated to this work from Griffith University - Whiplash Symposium 2017, 
Eurospine, Southern Denmark University, Institut Franco-Europeen de Chiropraxie, Karolinska Institutet, North American Spine Society, University of Quebec-Trois-Rivieres, University of Zurich, World Federation of Chiropractic; fees medical-legal expertise from the Canadian Chiropractic Protective Association. He is the Chair of the World Federation of Chiropractic Research Disability and Rehabilitation Committee. AB reports research grants unrelated to this work from University of Quebec-Trois-Rivieres, McGill University, Centre de recherche interdisciplinaire en réadaptation du Montréal métropolitain, Canadian Institutes of Health Research; fees medical-legal expertise from the Canadian Chiropractic Protective Association. GNK reports active research grants unrelated to this work from The Natural Sciences and Engineering Research, The National Institutes of Health, The Alberta Spine Foundation, The American Orthotic and Prosthetic Association, The New Frontiers in Research Fund and the Canadian Chiropractic Research Foundation. Travel expenditures unrelated to this work in the past year include Kiropraktik i Sverige Live, Et liv i bevegelse" (ELIB), the Nordic Institute of Chiropractic and Clinical Biomechanics, The American Chiropractic Association, The National Institutes of Health, The British Columbia Chiropractic Association, and The World Federation of Chiropractic. He is the Chair of the World Federation of Chiropractic Research Council. Fees for medical-legal expertise unrelated to this work from the Canadian Chiropractic Protective Association. JH reports that he holds multiple research grants from Danish and international funding agencies and charities. He has received coverage of travel expenditures from multiple sources internationally in connection with speaking engagements. Within the past year he has received speaking fees from Parker Seminars and Novartis. He is member of the World Federation of Chiropractic Research Council. SM reports research grants unrelated to this work from Ontario Chiropractic Association, Canadian Chiropractic Association, and Canadian Spinal Research Foundation. MS reports support from research grants unrelated to this work from the National Institutes of Health, Patient Centered Outcomes Research Institute, University of Pittsburgh and NCMIC Foundation; honoraria from NCMIC speaker's bureau; fees from medico-legal consulting services. JDC and CLY declare that they have no competing interests.

\section{Author details}

${ }^{1}$ Faculty of Health Sciences, Ontario Tech University, Oshawa, Canada. ${ }^{2}$ Centre for Disability Prevention and Rehabilitation at Ontario Tech University and CMCC, Oshawa, Canada. ${ }^{3}$ Division of Epidemiology, Dalla Lana School of Public Health, University of Toronto, Toronto, Canada.

${ }^{4}$ Département chiropratique, Université du Québec à Trois-Rivières, Trois-Rivières, Canada. ${ }^{5}$ School of Physical and Occupational Therapy, Faculty of Medicine McGill University, Montreal, Canada. ${ }^{6}$ Department of Sports Science and Clinical Biomechanics, University of Southern Denmark, Odense, Denmark. ${ }^{7}$ Nordic Institute of Chiropractic and Clinical Biomechanics, Odense, Denmark. ${ }^{8}$ Faculty of Rehabilitation Medicine, University of Alberta, Edmonton, Canada. ${ }^{9}$ Institute for Regional Health Research, University of Southern Denmark, Odense, Denmark. ${ }^{10}$ Canadian Memorial Chiropractic College, Toronto, Canada. ${ }^{11}$ School of Health and Rehabilitation Sciences, University of Pittsburgh, Pittsburgh, USA. ${ }^{12}$ Clinical and Translational Science Institute, University of Pittsburgh, Pittsburgh, USA.

Received: 6 April 2020 Accepted: 15 April 2020

Published online: 04 May 2020

\section{References}

1. World Health Organization. Q\&A on coronaviruses (COVID-19). 2020. https:// www.who.int/news-room/q-a-detail/q-a-coronaviruses.

2. World Health Organization. Coronavirus disease (COVID-19) advice for the public: Myth busters. 2020. https://www.who.int/emergencies/diseases/ novel-coronavirus-2019/advice-for-public/myth-busters.

3. World Federation of Chiropractic. Coronavirus Disease 2019 (COVID-19). Advice for chiropractors. 2020. http://www.wfc.org/website/images/wfc/ Latest_News_and_Features/Coronavirus_statement_2020_03_17.pdf.

4. International Chiropractors Association. Immune function and chiropractic what does the evidence provide? 2020. http://www.chiropractic.org/wpcontent/uploads/2020/03/ICA-Report-on-Immune-Function-andChiropractic-3-20-20.pdf.

5. International Chiropractors Association. Immune function and chiropractic what does the evidence provide? 2020. http://www.chiropractic.org/wpcontent/uploads/2020/03/Updated-Report-of-3-28-wtih-fixed-biblio.pdf.
6. Picchiottino M, Leboeuf-Yde C, Gagey O, Hallman DM. The acute effects of joint manipulative techniques on markers of autonomic nervous system activity: a systematic review and meta-analysis of randomized shamcontrolled trials. Chiropr Man Therap. 2019;27:17.

7. Goncalves G, Le Scanff C, Leboeuf-Yde C. Effect of chiropractic treatment on primary or early secondary prevention: a systematic review with a pedagogic approach. Chiropr Man Therap. 2018;26:10.

8. Clar C, Tsertsvadze A, I Court R, Hundt GL, Clarke A, Sutcliffe P. Clinical effectiveness of manual therapy for the management of musculoskeletal and non-musculoskeletal conditions: systematic review and update of UK evidence report. Chiropr Man Therap. 2014;22:12.

9. Lilienfeld SO, Ammirati R, David M. Distinguishing science from pseudoscience in school psychology: science and scientific thinking as safeguards against human error. J Sch Psychol. 2012;50:7-36.

10. Teodorczyk-Injeyan JA, et al. Elevated production of nociceptive CC chemokines and sE-selectin in patients with low back pain and the effects of spinal manipulation: a nonrandomized clinical trial. Clin J Pain. 2018;34: 68-75.

11. Teodorczyk-Injeyan JA, Injeyan HS, Ruegg R. Spinal manipulative therapy reduces inflammatory cytokines but not substance P production in normal subjects. J Manip Physiol Ther. 2006;29:14-21.

12. Peters $\mathrm{DH}$, Tran NT, Adam T. Implementation research in health: a practical guide. Alliance for Health Policy and Systems Research, World Health Organization; 2013.

13. White Junod. FDA and clinical drug trials: a short history. 2020. https://www. fda.gov/media/110437/download.

14. World Health Organization, 2020. Coronavirus disease (COVID-19) technical guidance: patient management. 2020. https://www.who.int/emergencies/ diseases/novel-coronavirus-2019/technical-guidance/patient-management.

\section{Publisher's Note}

Springer Nature remains neutral with regard to jurisdictional claims in published maps and institutional affiliations.

\section{Ready to submit your research? Choose BMC and benefit from:}

- fast, convenient online submission

- thorough peer review by experienced researchers in your field

- rapid publication on acceptance

- support for research data, including large and complex data types

- gold Open Access which fosters wider collaboration and increased citations

- maximum visibility for your research: over $100 \mathrm{M}$ website views per year

At BMC, research is always in progress.

Learn more biomedcentral.com/submissions 